Schmerz 2022 $\cdot 36: 363-370$

https://doi.org/10.1007/s00482-021-00612-y

Angenommen: 9. November 2021

Online publiziert: 16. Dezember 2021

(c) The Author(s), under exclusive licence to Springer Medizin Verlag GmbH, ein Teil von Springer Nature 2021, korrigierte Publikation 2022
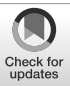

\section{Vorstellung eines innovativen interdisziplinären, sektoren- übergreifenden und gestuften Behandlungskonzepts für Schmerzpatient*innen im Rahmen eines regionalen Selektivvertrages nach §140a SGB V}

\author{
Hubertus Kayser ${ }^{1}$. Nadine Schneider ${ }^{1}$. Guido Schmiemann ${ }^{2}$ \\ ${ }^{1}$ Abteilung Schmerzmedizin, Paracelsus-Klinik Bremen, Bremen, Deutschland \\ ${ }^{2}$ Institut für Public Health und Pflegeforschung (IPP), Abteilung Versorgungsforschung, Universität \\ Bremen, Bremen, Deutschland
}

\section{Zusammenfassung}

Hintergrund: Eine gestufte Versorgung in der Behandlung chronisch schmerzkranker Patient*innen ist in Deutschland nur in Ansätzen vorhanden. Vor dem Hintergrund der aktuellen Pandemiebedingungen hat sich eine Unter- und Fehlversorgung weiter verschärft.

Ziel: Entwicklung und Aufbau eines sektorenübergreifenden Behandlungskonzepts für schmerzkranke Patient*innen im Rahmen eines Selektivvertrags.

Methoden: Eingebettet in bereits vorhandene Versorgungsstrukturen wurden nach vorbezeichneten Kriterien sieben Versorgungspfade (Clinical Pathways, CP) definiert, in die eingeschriebene Patient ${ }^{*}$ innen nach einem interdisziplinären Assessment geleitet werden.

Aufbau: Im CP I verbleiben die Patient*innen in der Regelversorgung. Im CP II wird zusätzlich eine einmalige interprofessionelle Edukation von $3 \mathrm{~h}$ zur Prophylaxe weiterer Chronifizierung angeboten. Im CP III gehen die Patient*innen für sechs Monate in eine fachärztlich spezialisierte ambulante Schmerzbehandlung. Das CP IV ist eine teilstationäre multimodale Therapie, bei der viele Präsenztage durch telemedizinische Inhalte mit Unterstützung einer Reha-App ersetzt werden. CP V und VI sind vollstationäre multimodale Behandlungen über acht und 15 Tage. In CP VII kann bei ambulantem psychotherapeutischen Behandlungsbedarf für Patient*innen nach vollstationärer Behandlung zur Überbrückung des Wartezeitraums über sechs Monate eine niederfrequente Psychotherapie erfolgen.

Qualitätssicherung und Evaluation: Die wissenschaftliche Begleitung erfolgt mittels ausgewählter Fragebögen und psychometrischer Testverfahren jeweils drei, sechs und zwölf Monate nach erfolgtem Assessment, um die Inhalte des Vertrags zu evaluieren.

\section{Schlüsselwörter}

Gestufte Versorgung von Schmerzpatient*innen · Interdisziplinäres Assessment · Teilstationäre Therapie · Telemedizinische Inhalte · Psychotherapeutische Nachbehandlung 


\section{Zulassungskriterien IDA I und IDA II}

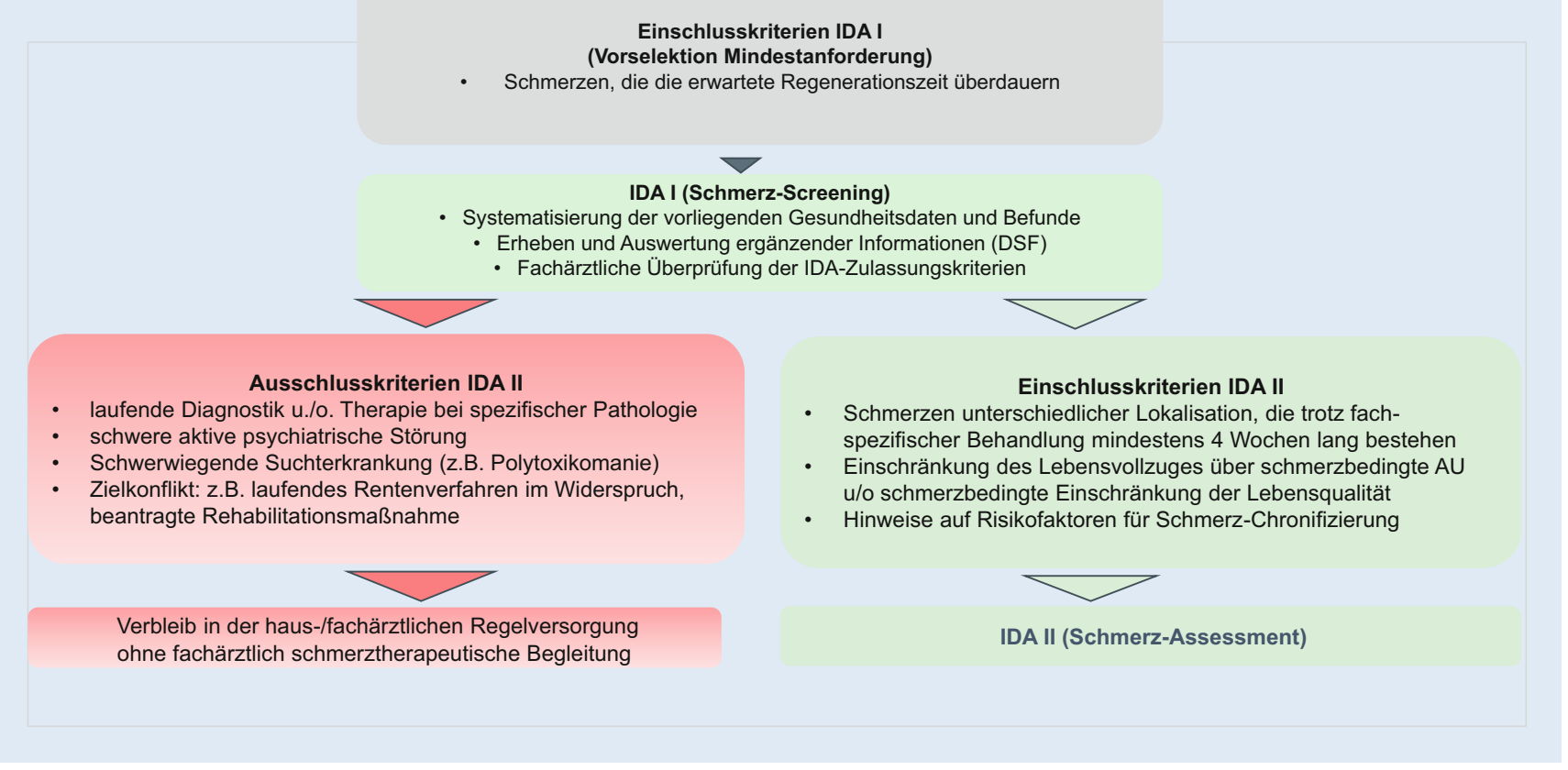

Abb. 1 \ Zulassungskriterien Interdisziplinäres Assessment IDA I und IDA II. (Eigene Darstellung). DSF Deutscher Schmerzfragebogen, $A \cup$ Arbeitsunfähigkeit

Zur Verbesserung der Versorgung von Menschen mit chronischen Schmerzen haben die Paracelsus-Klinik Bremen und die regionale Krankenkasse AOK Bremen/Bremerhaven im Rahmen der AOKReihe "Der kurze Weg" ein sektorenübergreifendes Behandlungskonzept initiiert. Eingebettet in bereits vorhandene Versorgungsstrukturen, wie z.B. die ambulante Behandlung im Rahmen der Qualitätssicherungsvereinbarung Schmerztherapie der KBV [12] oder die stationäre multimodale Schmerztherapie nach Operationen- und Prozedurenschlüssel OPS 8-918, werden abgestuft insgesamt sieben Versorgungspfade (Clinical Pathways, CP) definiert, in die Patient*innen nach einem interprofessionellen Assessment geleitet werden. Diese bieten verschiedene Innovationen, die die Nachhaltigkeit der schmerztherapeutischen Interventionen unterstützen sollen.

\section{Einleitung und Ziele}

Anlass und Hintergrund der Erarbeitung eines Selektivvertrags waren Berichte über die Unterversorgung chronisch schmerzkranker Menschen, die sich durch die aktu- elle Pandemie weiter verschärft hat $[2,6,8$, 16]. Bekannt ist zusätzlich eine mangelnde Stufung der Diagnostik und Therapie der Schmerzmedizin in Deutschland [13, $15,19,25]$, die zu weiterer Unter- und Fehlversorgung mit langen Wartezeiten und damit verbundener weiterer Chronifizierung der Patient*innen mit immensen Kosten für das Gesundheitswesen führt [1, $14,21,22,24]$. Zum Beispiel wird für die Behandlung von Rückenschmerzen demnach eine multimodale Schmerztherapie zumindest teilstationär gefordert [21, 22]. Außerdem wird angemerkt, dass "einer bedarfsgerechten sektorenübergreifenden Versorgung in erster Linie die starke Abschottung der einzelnen Leistungssektoren entgegensteht" und deshalb eine Steuerung von Patient*innenwegen zum Patient*innenwohl empfohlen wird [22].

Im Rahmen eines Selektivvertrags nach §140a SGB V „Besondere Versorgung" wurde ein sektorenübergreifendes Behandlungskonzept zwischen der Paracelsus-Klinik Bremen, dem dort angeschlossenen medizinischen Versorgungszentrum (MVZ) und der Krankenkasse AOK Bremen/Bremerhaven im Rahmen eines Kompetenznetzwerks erarbeitet, um die
Behandlung von Schmerzpatient*innen zu verbessern. Die Paracelsus-Klinik Bremen ist ein Anbieter für ambulante speziell schmerztherapeutische Behandlung und vollstationäre multimodale Schmerztherapie.

Der Vertrag ermöglichteine Versorgung und Betreuung von Betroffenen, die deutlich über die reguläre Versorgung hinausgeht, aber andererseits auch in vorhandene Strukturen eingebettet ist. Darüber hinaus soll durch ein gestuftes Konzept eine Steuerung zum Patient*innenwohl erfolgen [9, 11, 22]. Eine bedarfsorientierte Steuerung zeigte in der Behandlung von Rückenschmerzen bereits positive Ergebnisse in Bezug auf schmerzbezogene, psychometrische und soziale Kennwerte [3]. Der vorliegende Vertrag adressiert neben Patient*innen mit Rückenschmerzen aber auch alle anderen Schmerzpatient*innen, die sich in der Abteilung Schmerzmedizin anmelden und sich in den Vertrag einschreiben. Im Rahmen des Vertrags werden sowohl das Assessment als auch die Edukation im ambulanten Bereich, die ambulante schmerzmedizinische Versorgung und die (teil)stationäre multimodale Schmerz- 


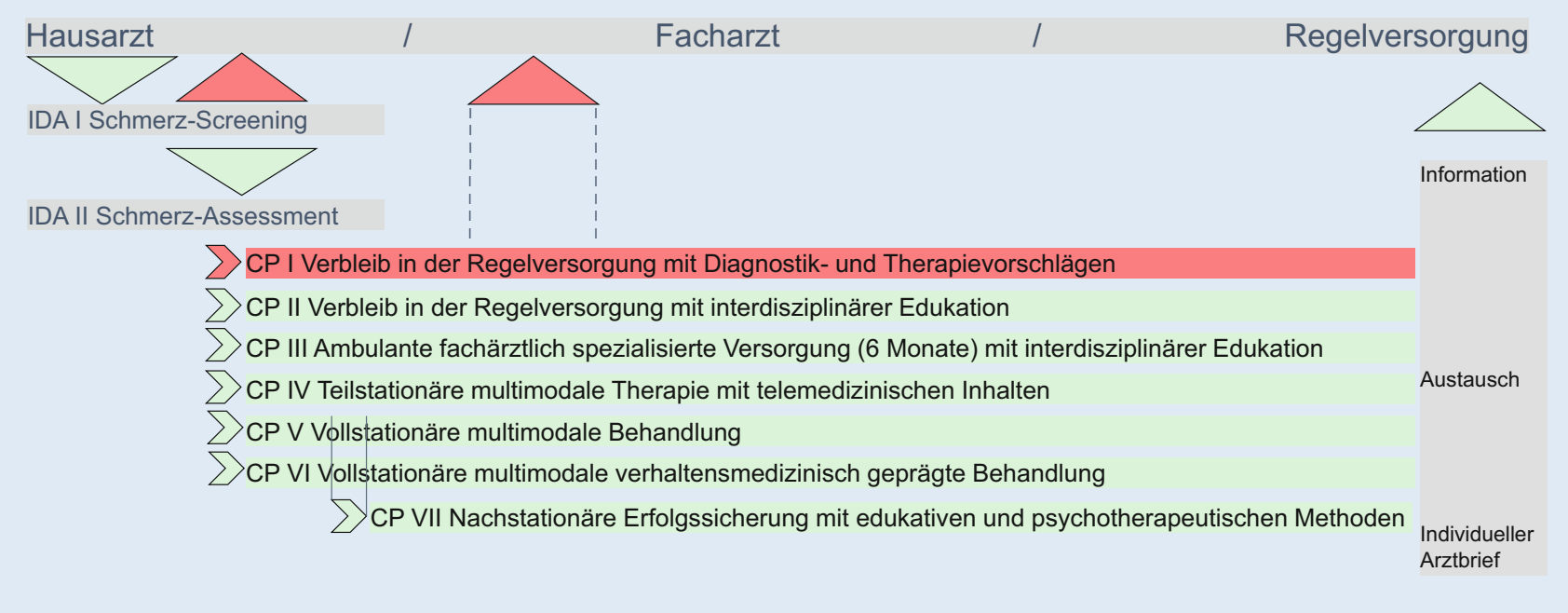

Abb. 2 \ Steuerung schmerzkranker Patient*innen mit systematischen Einschlusskriterien in ein abgestimmtes Behandlungssetting und Zuordnung in die Clinical Pathways $(C P) I-V I I$ oder in eine andere notwendige Diagnostik/Therapie. (Eigene Darstellung). IDA Interdisziplinäres Assessment

behandlung im Rahmen der Versorgungspfade gesondert vergütet.

\section{Methoden und Aufbau}

\section{Interdisziplinäres Assessment (IDA I und II)}

Zentrale Steuerungsfunktion übernimmt das interdisziplinäre Assessment (IDA), das sich in IDA I und IDA II aufteilt (• Abb. 1).

IDA I ist eine ärztliche Gesamtsicht auf die bisherige Krankheitsgeschichte mit Zusammenstellung aller relevanten Diagnosen, Befunde und durchgeführten Therapien ohne direkten Patientenkontakt. Es findet in der Klinik nach Eingang des Deutschen Schmerzfragebogens (DSF) und der relevanten Vorbefunde statt.

\section{Für das Schmerzassessment IDA I gelten folgende Prüfelemente}

- Ausschluss von Red Flags, Ermittlung von Yellow Flags (siehe IDA II)

- Ermittlung des Chronifizierungsgrads, auch unter Nutzung von Fragebögen und Kontaktaufnahme zu Vorbehandelnden

- Prüfung der IDA-II-Zulassungsvoraussetzungen. Neben der Sichtung und Aufbereitung der relevanten Gesundheitsdaten sind die fachärztliche Sichtung und Bewertung der vorlie- genden Befunde und die Erstellung eines Kurzberichts obligat.

IDA II stellt eine von den zu empfehlenden Therapien unabhängige interdisziplinäre Untersuchung und Befunderhebung dar. Es unterscheidet sich grundlegend von dem OPS 1-910 der Krankenhausregelversorgung, der hohe Eingangsvoraussetzungen bei den Patienten fordert und somit bereits eine hohe Chronifizierung zur Voraussetzung hat.

\section{Für das Schmerzassessment IDA II gelten folgende Prüfelemente}

- Ausschluss von Red Flags, Ermittlung und Bewertung von Yellow Flags (psychosoziale Risikofaktoren für eine Chronifizierung von Schmerzen wie Katastrophisieren, Angst-Vermeidungsverhalten oder dysfunktionales Durchhalteverhalten, für die neben dem DSF zusätzliche Instrumente eingesetzt werden können, wie z.B. die Pain Catastrophizing Scale (vgl. hierzu [11]) und der Avoidance-Endurance Fast Screen (AE-FS) [26]).

- Wird durch ein interprofessionelles Team gemeinsam erbracht und umfasst die schmerzmedizinische, körperlichfunktionelle und psychotherapeutische Diagnostik

- Je nach spezifischer Indikationsstellung kann zusätzlich auf Fachärzt*innen aus den Fachdisziplinen Orthopäde, Neurochirurgie und Neurologie zurückgegriffen werden

- Als Ergebnis steht eine im interdisziplinären Team entwickelte dokumentierte Empfehlung zur weitergehenden Diagnostik und Therapie in einem individueller Arztbrief (• Abb. 2)

\section{Clinical Pathway (CP) I}

Verbleib in der ambulanten Regelversorgung

Indikationen

- Neu aufgetretene Akutschmerzsymptomatik

- Chronifizierungsgrad I (Mainz Pain Staging System MPSS) mit und ohne Yellow Flags

\section{Leistungen}

- Der Arzt/Hausarzt (HA)/Facharzt (FA) erhält nach dem Schmerzassessment (IDA II) Diagnostik- und Therapievorschläge für die weitere Schmerzbehandlung analog eines Zweitmeinungsverfahrens (Medikation, Heilmittel u.a.)

Leistungen aus dem Vertrag enden hier. 


\section{Clinical Pathway (CP) II}

Verbleib in der Regelversorgung Angebot einer interdisziplinären Edukation

Indikationen

- Neu aufgetretene Akutschmerzsymptomatik

- Chronifizierungsgrad I/II (MPSS) mit Yellow Flags

- Monomodale Therapie noch ausreichend

\section{Leistungen}

- Therapieentscheidung: Beratung über Ergebnis der Untersuchungen und Besprechen des individuellen Risikoprofils mit dem Hinweis auf Möglichkeiten mittels gemeinsamer Entscheidungsfindung (Shared Decision-Making)

- Der Hausarzt (HA)/Facharzt (FA) erhält nach dem Schmerzassessment (IDA II) Therapievorschläge für die weitere Schmerzbehandlung analog eines Zweitmeinungsverfahrens (Medikation, Heilmittel u. a.)

- Darüber hinaus erhalten die Patient*innen die Möglichkeit, an einem einmaligen interdisziplinären Edukationsmodul in der Gruppe zur Vermittlung von Copingstrategien teilzunehmen (Gruppengröße bis 12 Teilnehmende)

\section{Clinical Pathway (CP) III}

Besondere ambulante fachärztlich spezialisierte Versorgung über sechs Monate innerhalb des Selektivvertrags

\section{Indikationen}

- Überwiegend Chronifizierungsgrad I/II (MPSS), Schweregrad der Schmerzerkrankung 1-2 (nach von Korff)

- Schmerzdauer: Übergang akut/ subakut; $>4$ Wochen

- Hausärztliche Versorgung der Schmerzerkrankung ausgeschöpft und schmerzmedizinische Expertise notwendig

- Mittleres bis hohes Chronifizierungsrisiko (nach psychosozialer Anamnese)

- Schwierigere medikamentöse Einstellung oder ambulanter Medikamen- tenentzug möglich und geboten (z. B. Nichtopioide)

- Schmerzmedizinische Patientenführung notwendig

\section{Leistungen}

- Jeweils Erhebung der standardisierten Schmerzanamnese, Schmerzanalyse, Überprüfung der Medikation, differenzialdiagnostische Abklärung, standardisierte Dokumentation und Bericht an den HA/FA

- Schneller Zugang zum und Beschleunigung der Diagnostik und Behandlung im Netzwerk der Paracelsus-Klinik Bremen

- Stabilisierung der Patientin/des Patienten; möglichst schnelle Rückführung in die hausärztliche Behandlung mit Angebot einer interdisziplinären Edukation zur Vermittlung von Copingstrategien

- Nach sechs Monaten Behandlung innerhalb des Selektivvertrags Überprüfung, ob Weiterbehandlung beim überweisenden HA/FA möglich oder eine weitere schmerztherapeutische Behandlung im Rahmen der Regelversorgung notwendig ist

- Therapieentscheidung: Beratung über Ergebnis der Untersuchungen und Besprechen des individuellen Risikoprofils mit dem Hinweis auf Möglichkeiten mittels gemeinsamer Entscheidungsfindung (Shared Decision-Making)

\section{Clinical Pathway (CP) IV}

Teilstationäre multimodale Behandlung (15 Werktage)

\section{Indikationen}

- Schmerzdauer: $>8$ Wochen

- Überwiegend Chronifizierungsgrad II/III (MPSS), Schweregrad der Schmerzerkrankung 2-4 (nach von Korff)

- Schmerzerkrankung ambulant fachärztlich nicht beherrschbar

- Yellow Flags

- Im Wesentlichen schmerzrelevante stabile komplexe Komorbidität

\section{Leistungen}

- Drei Wochen Behandlung mit Präsenztagen und telemedizinischen Einheiten nach einem festgelegten Behandlungsplan (ca. $100 \mathrm{TE}$ ) mit ärztlicher Behandlungsleitung und sozialmedizinischer Begleitung, wöchentlichen Teamvisiten und Organisation der Weiterversorgung

- Die Patient*innen erhalten ein OnlineBenutzerkonto mit einem individuellen, multimodalen Therapieplan. Die Patient*innen werden bei der Übungsdurchführung durch ein Online-Tool begleitet, das Feedback und Aktivität direkt an die Therapeuten weiterleitet.

- Es handelt sich um eine koordinierte und gruppenbasierte teilstationäre Behandlung (max. acht Patient*innen).

\section{Clinical Pathway (CP) V}

Vollstationäre Behandlung: acht Tage interdisziplinäre multimodale Schmerztherapie (IMST)

\section{Indikationen}

- Schmerzdauer: > 8 Wochen

- Überwiegend Chronifizierungsgrad II/III (MPSS), Schweregrad der Schmerzerkrankung 3-4 (nach von Korff)

- Schmerzerkrankung ambulant fachärztlich nicht beherrschbar

- Relevante, potenziell destabilisierende somatische Komorbidität oder Multimorbidität

- Schmerzrelevante psychisch stabile komplexe Komorbidität

- Analgetikafehlgebrauch

- Ggf. erhebliche Funktionseinschränkungen mit unzureichender Mobilität

Leistungen: koordiniert gruppenbasierte stationäre IMST mit max. acht $\mathrm{Pa}$ tient*innen analog OPS 8-918.02.

\section{Clinical Pathway (CP) VI}

Vollstationäre Behandlung: 15 Tage interdisziplinäre multimodale Schmerztherapie (IMST)

Indikationen

- Schmerzdauer: $>8$ Wochen

- Überwiegend Chronifizierungsgrad II/III (MPSS), Schweregrad der Schmerzerkrankung 3-4 (nach von Korff) 
Tab. 1 Patient*innenenkriterien - Ergebnis interdisziplinäres Assessment (IDAII); mod. nach [18] Patientenkriterien - Ergebnis interdisziplinäres Assessment (IDA II)

\begin{tabular}{|c|c|c|c|c|}
\hline Kriterium & $\begin{array}{l}\text { Mögliche Instrumen- } \\
\text { te }\end{array}$ & $\begin{array}{l}\text { Ambulante ST } \\
\text { (CP I-III) }\end{array}$ & $\begin{array}{l}\text { Teilstationäre IMST } \\
\text { (CP IV) }\end{array}$ & $\begin{array}{l}\text { Stationäre IMST } \\
\text { (CP V-VI) }\end{array}$ \\
\hline Schmerzdauer & - & $\begin{array}{l}\text { Übergang akut/subakut } \\
>4 \text { Wochen }\end{array}$ & $>8$ Wochen & $>8$ Wochen \\
\hline $\begin{array}{l}\text { Chronifizierungsstadium } \\
\text { Schmerzgraduierung }\end{array}$ & $\begin{array}{l}\text { MPSS } \\
\text { von Korff }\end{array}$ & $\begin{array}{l}\text { MPSS I-II } \\
\text { von Korff 1-2 }\end{array}$ & $\begin{array}{l}\text { MPSS II-III } \\
\text { von Korff 2-4 }\end{array}$ & $\begin{array}{l}\text { MPSS II-III } \\
\text { von Korff 3-4 }\end{array}$ \\
\hline Psychosoziale Faktoren & Lt. Assessment & $\begin{array}{l}\text { Schmerzrelevante Risiko- } \\
\text { faktoren }\end{array}$ & $\begin{array}{l}\text { Ausgeprägt/ } \\
\text { schmerzrelevant/ } \\
\text { aufrechterhaltend }\end{array}$ & Manifest/schmerzrelevant \\
\hline Chronifizierung & - & $\begin{array}{l}\text { Mittleres bis hohes Risi- } \\
\text { ko (nach psychosozialer } \\
\text { Anamnese) }\end{array}$ & $\begin{array}{l}\text { Fortgeschrittene/ } \\
\text { bereits eingetretene } \\
\text { Chronifizierung }\end{array}$ & $\begin{array}{l}\text { Fortgeschrittene/ } \\
\text { bereits eingetretene } \\
\text { Chronifizierung }\end{array}$ \\
\hline Arbeitsunfähigkeit & - & $\begin{array}{l}\text { Nicht mehr als } \\
6 \text { Wochen }\end{array}$ & $>6$ Wochen & $>6$ Wochen \\
\hline $\begin{array}{l}\text { Komorbidität somatisch/ } \\
\text { psychisch }\end{array}$ & - & $\begin{array}{l}\text { Stabile Situation, } \\
\text { keine komplexe } \\
\text { schmerzrelevante } \\
\text { Komorbidität }\end{array}$ & $\begin{array}{l}\text { Im Wesentlichen } \\
\text { stabile komplexe } \\
\text { Komorbidität }\end{array}$ & $\begin{array}{l}\text { Relevante, potenziell } \\
\text { destabilisierende } \\
\text { Komorbiditäten } \\
\text { (CP V: eher somatisch, CP Vl: eher } \\
\text { psychisch) }\end{array}$ \\
\hline $\begin{array}{l}\text { Inanspruchnahme von medi- } \\
\text { zinischen Leistungen } \\
\text { (Behandlungsdauer, Art) }\end{array}$ & $\begin{array}{l}\text { Leistungsausdruck } \\
\text { Krankenkasse }\end{array}$ & $\begin{array}{l}\text { Nicht zwingend } \\
\text { hoch }\end{array}$ & Eher hoch & Eher hoch \\
\hline $\begin{array}{l}\text { Beeinträchtigung Therapie- } \\
\text { ergebnis durch Wegezeit } \\
\text { möglich? }\end{array}$ & - & Nicht relevant & $\mathrm{Ja}$ & Nein \\
\hline Mobilität & - & $\begin{array}{l}\text { Eigenständige } \\
\text { Anreise möglich }\end{array}$ & $\begin{array}{l}\text { Eigenständige } \\
\text { Anreise möglich }\end{array}$ & $\begin{array}{l}\text { Eingeschränkt (kein Ausschlusskri- } \\
\text { terium) (CPV) }\end{array}$ \\
\hline
\end{tabular}

Bemerkung: Schmerzdauer: Grenzen sind fließend, Psychosoziale Faktoren und Chronifizierung: Überlappungen möglich bei unscharfen Kriterien, Abgrenzung ambulant/stationär: nach § 39(1) SGB V (vgl. [18])

IDA Interdisziplinäres Assessment, MPSS Mainz Pain Staging System, von Korff Schweregrad der Schmerzerkrankung nach von Korff, IMST interdisziplinäre multimodale Schmerztherapie, ST Schmerztherapie, CP Clinical Pathway

- Schmerzerkrankung ambulant fachärztlich nicht beherrschbar

- Schmerzbedingter Abbruch einer Rehabilitationsbehandlung bzw. nach Rehabilitation schmerzbedingt nicht arbeitsfähig

- Relevante, potenziell destabilisierende psychische Erkrankung, die eine höherfrequente psychotherapeutische Mitbehandlung erfordert

- Ggf. Schmerzmittelabhängigkeit/missbrauch und Entzugsbehandlung von Analgetika

- Ggf. fehlgeschlagener ambulanter Medikamentenentzug bzw. bei Rückfall

- Ggf. schlechte häusliche Versorgung oder hohe Belastung im häuslichen Umfeld

Leistungen: koordiniert gruppenbasierte stationäre IMST mit max. acht Patient*innen analog OPS 8-918.14.

\section{Clinical Pathway (CP) VII}

Nachstationäre Therapieerfolgssicherung

Indikationen

- Ambulanter, psychotherapeutischer Behandlungsbedarf zur Sicherung des Therapieerfolgs nach stationärer Versorgung (CP V, VI)

- Überbrückung des Zeitraums bis zur Übergabe in die ambulante psychotherapeutische Regelversorgung

- Verhinderung stationärer und teilstationärer psychiatrischer Behandlungsnotwendigkeit

\section{Leistungen}

- Sechs Monate Therapieerfolgssicherung bis zur ambulanten psychotherapeutischen Regelversorgung in maximal vier niederfrequenten Ein- zelsitzungen (eine Therapieeinheit umfasst mindestens $40 \mathrm{~min}$ )

- Überprüfung und Etablierung der Transferprozesse der stationären klinischen Erfolge in den häuslichen Alltag durch edukative und psychotherapeutische Methoden

\section{Struktur- und Prozessparameter}

Bei der Erstellung der Patient*innenselektion haben wir uns an den Empfehlungen zu Struktur- und Prozessparametern der Adhoc-Kommission "Interdisziplinäre Multimodale Schmerztherapie" der Deutschen Schmerzgesellschaft e.V. [18] orientiert. Diese Empfehlungen bieten die Grundlage zur Weiterentwicklung der interdisziplinären multimodalen Schmerztherapie in allen Sektoren (- Tab. 1, 2 und 3). 


\section{Übersichten}

\begin{tabular}{|c|c|c|c|c|}
\hline \multicolumn{5}{|l|}{ Prozesskriterien } \\
\hline Kriterium & $\begin{array}{l}\text { Ambulante ST } \\
\text { (CP I-III) }\end{array}$ & $\begin{array}{l}\text { Teilstationäre. IMST } \\
\text { (CP IV) }\end{array}$ & $\begin{array}{l}\text { Stationäre } \\
\text { IMST } \\
\text { (CP V-VI) }\end{array}$ & Kommentar \\
\hline $\begin{array}{l}\text { Berufs-bzw. alltags- } \\
\text { begleitende Therapie } \\
\text { möglich? }\end{array}$ & $\mathrm{Ja}$ & Eher nein & Nein & $\begin{array}{l}\text { In der TS IMST ist eine berufsbegleitende } \\
\text { Behandlung nicht möglich, jedoch eine } \\
\text { alltagsbegleitende }\end{array}$ \\
\hline $\begin{array}{l}\text { Frequenz/Intensität } \\
\text { der Behandlung }\end{array}$ & $\begin{array}{l}\text { Niedrigschwelliges und } \\
\text { ggf. berufsbegleitendes } \\
\text { Programm }\end{array}$ & $\begin{array}{l}\text { Hochintensiv (100 TE grup- } \\
\text { pen- und überwiegend tele- } \\
\text { medizinbasiert) }\end{array}$ & $\begin{array}{l}\text { Hochintensiv; } \\
\text { gruppenba- } \\
\text { siert }\end{array}$ & - \\
\hline $\begin{array}{l}\text { Festgelegter Behand- } \\
\text { lungsplan }\end{array}$ & Ja & Ja & $\mathrm{Ja}$ & Grundbedingung nach SVR-GA \\
\hline Zielsetzung & $\begin{array}{l}\text { Chronifizierung aufhal- } \\
\text { ten/verhindern }\end{array}$ & "Functional restoration" & $\begin{array}{l}\text { "Functional } \\
\text { restoration" }\end{array}$ & - \\
\hline
\end{tabular}

\begin{tabular}{|l|l|l|l|}
\hline \multicolumn{2}{|l|}{ Tab. 3 Medikamentöse Einstellung. (Modifiziert nach [18]) } \\
\hline Medikamentöse Einstellung & $\begin{array}{l}\text { Tmbulante ST } \\
\text { (CP I-III) }\end{array}$ & $\begin{array}{l}\text { Teilstationäre. IMST } \\
\text { (CP IV) }\end{array}$ & $\begin{array}{l}\text { Stationäre IMST } \\
\text { (CP V-VI) }\end{array}$ \\
\hline Kriterium & $\begin{array}{l}\text { Unproblematische medikamen- } \\
\text { tös } \\
\text { analgetische Behandlung }\end{array}$ & Polypharmazie & Polypharmazie \\
\hline Analgetika & $\begin{array}{l}\text { Kein weiterer medizinischer } \\
\text { Handlungsbedarf zur Verbes- } \\
\text { serung der Schmerzsituation }\end{array}$ & $\begin{array}{l}\text { Komplexe Situation (Interaktion, } \\
\text { Polypharmazie usw.) }\end{array}$ & $\begin{array}{l}\text { Komplexere Situation (Interaktion, Polypharmazie } \\
\text { usw.) }\end{array}$ \\
\hline $\begin{array}{l}\text { Weiterer medizinischer } \\
\text { mit Einfluss auf den } \\
\text { Schmerz }\end{array}$ & $\begin{array}{l}\text { Nicht bei Fehlgebrauch von } \\
\text { Opioiden und/oder Cannabi- } \\
\text { noiden }\end{array}$ & - & $\begin{array}{l}\text { Bei Fehlgebrauch von Opioiden und/oder Cannabi- } \\
\text { noiden }\end{array}$ \\
\hline Fehlgebrauch & $\begin{array}{l}\text { Ja: bei Fehlgebrauch von Nicht- } \\
\text { opioiden } \\
\text { Nein: bei Fehlgebrauch zen- } \\
\text { tralwirksamer (Co-)Analgetika }\end{array}$ & $\begin{array}{l}\text { Ggf.: bei Fehlgebrauch von } \\
\text { Nichtopioiden } \\
\text { Ggf.: bei Fehlgebrauch zentral- } \\
\text { wirksamer (Co-)Analgetika }\end{array}$ & $\begin{array}{l}\text { Bei komplexer Situation stationär durchzuführen, } \\
\text { u. a. auch bei relevanter psychischer Komorbidität } \\
\text { oder Scheitern eines ambulanten Entzugs bzw. bei } \\
\text { Rückfall }\end{array}$ \\
\hline Medikamentenentzug
\end{tabular}

\section{Qualitätssicherung und Evaluation}

Um die Qualitätssicherung sicherzustellen, sind vonseiten der Leistungserbringer eine Kooperation bezüglich der Inhalte und Ziele der Behandlung, eine Koordination der unterschiedlichen Aktivitäten und Disziplinen sowie eine effiziente Kommunikation über die jeweils erfolgte Diagnostik und Therapie notwendig. Wesentliche Elemente stellen in diesem Rahmen auch der Informationstransfer sowie die gemeinsame Evaluation dar. Grundlagen der QMSysteme sind die GBA-Richtlinie, das Patientenrechtegesetz sowie das Regelwerk nach DIN EN ISO 9001.

Neben der bestehenden hausinternen Qualitätssicherung sollen auch die klinischen Effekte der Intervention syste- matisch erfasst werden. Geplant sind die Auswertung der vonseiten der beteiligten Krankenkasse routinemäßig erhobenen Daten sowie zusätzlich individueller Assessmentergebnisse und ihrer Veränderung im längsschnittlichen Verlauf.

Durch die Auswertung der Routinedaten können beispielsweise über die Teilnahme am Programm hinausgehende Angaben gemacht werden zu:

- Dauer der Arbeitsunfähigkeitszeiten im Verlauf,

- Verordnungskosten (Medikation),

- Anzahl und Fachrichtung der Behandler.

Diese Routinedaten werden allerdings nur für die Versicherten der beteiligten Krankenkasse vorliegen.
Darüber hinaus werden die eingesetzten Assessmentinstrumente (Deutscher Schmerzfragebogen DSF mit Schmerzbeschreibungsliste SBL, Schweregrad der Schmerzerkrankung nach von Korff, Depression, Anxiety and Stress Scale DASS, Marburger Fragebogen zum habituellen Wohlbefinden Fw7 [MFHW], Einschränkungen in der gesundheitsbezogenen Lebensqualität VR-12, Quality of Life Impairment by Pain Inventory QLIP) [7] und das Mainz Pain Staging System MPSS vollständig elektronisch via Tablet erhoben. Dies gilt inzwischen auch für die übrigen Patient*innen der Abteilung, sodass perspektivisch nicht nur die Effekte für die Teilnehmer*innen am Selektivvertrag, sondern darüber hinaus auch vergleichen- 
de Evaluationen zur "Usual Care"-Gruppe ermöglicht werden.

Ein positives Votum der Ethikkommission vorausgesetzt wird diese Erhebung perspektivisch auch den Vergleich von Teilnehmenden/Nichtteilnehmenden ermöglichen, um dadurch auch krankheitsbezogene Effekte der Intervention ermitteln zu können. Für die längsschnittliche Beobachtung der Effekte ist eine Erhebung drei, sechs und zwölf Monate nach erfolgtem IDA II resp. Erstkontakt in der Ambulanz mithilfe des Schmerz-Verlaufsfragebogens geplant.

\section{Diskussion}

Vorliegende Arbeit beschreibt ein sektorenübergreifendes Behandlungskonzept, das seit dem 01.01.2021 an den Start gegangen ist. Es bietet im Rahmen der Vorgaben der Ad-hoc-Kommission „Interdisziplinäre Multimodale Schmerzthera-

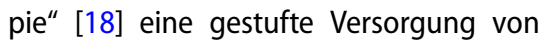
Schmerzpatient*innen, die in der Folge evaluiert wird.

In diesem Konzept finden sich aber auch praxisnah einige Neuerungen:

- Bei entsprechender Voraussetzung, die durch ein interdisziplinäres Assessment (IDA I und II) erhoben wird, bieten wir im CP II und III ein interdisziplinäres Edukationsmodul in der Gruppe an. Ähnlich wie bei dem E-IMST-Modul in PAIN 2020 [11] handelt es sich um einen einmaligen Termin, der interprofessionell bei einer maximalen Gruppengröße von zwölf Patient*innen z. B. das bio-psycho-soziale Schmerzmodell und Selbstmanagement-Optionen vermittelt.

- Entsprechend den Empfehlungen der Nationalen Versorgungsleitlinie Kreuzschmerz [4] bedarf es zusätzlich zur bereits bestehenden Versorgung mit stationären bzw. teilstationären interdisziplinären multimodalen Angeboten auch niederschwelliger Ansätze. Insbesondere bei der frühzeitigen Identifizierung von Patient*innen zu Beginn des Chronifizierungsprozesses sind die intensiven Formen der Behandlung oftmals nicht notwendig. In diesen Fällen ist es in CP III im Netzwerk des Krankenhauses erstmals möglich, außerhalb des
Einheitlichen Bewertungsmaßstabs (EBM) ambulante fachärztlich spezialisierte Schmerztherapie für diese Patient*innen über sechs Monate anzubieten. Auch hier wird ein interdisziplinäres Edukationsmodul für die Patient*innen vorgehalten.

- Im CP IV erfolgt eine teilstationäre multimodale Behandlung über drei Wochen im Rahmen eines koordinierten gruppenbasierten Programms mit maximal acht Patient*innen. Ein großer Anteil des Programms findet bei nur sieben Präsenztagen Telemedizin-basiert statt [17]. Die Visiten führen wir via Videosprechstunde durch, während Edukationen (z. B. Schmerzbewältigung, Entspannung) und Physiotherapie im LiveChat im gruppenbasierten Setting stattfinden. Ergänzt wird dieses Setting durch den Einsatz einer zertifizierten Online-Plattform. App-basierte Behandlungen chronisch unspezifischer Rückenschmerzen sind national und international bereits untersucht [20, 23]. Mithilfe der Software können medizinische Einrichtungen mit ihren Patient*innen Therapiemaßnahmen - Übungen, Seminare, Schulungen und Vorträge - online durchführen, unabhängig von Zeit und Ort. In der Rehabilitation werden entsprechende Tools für die Nachsorge bei Erkrankungen aus dem orthopädischen Fachgebiet als gleichwertig zur IRENAMaßnahme angesehen [10].

Durch das Einbeziehen moderner Kommunikationsmittel wie Videosprechstunde, Patientenportal und Therapie-Apps kann eine qualitativ hochwertige Schmerztherapie auch Patient*innen zuteilwerden, für die eine stationäre Behandlung aufgrund der aktuellen Lebenssituation oder auch in Zeiten der Pandemie nicht in Frage kommt. Durch den direkten Informationsaustausch soll die Compliance gefördert und der nachhaltige Therapieerfolg gesichert werden.

- Eine weitere Neuerung umfasst die nachstationäre Therapieerfolgssicherung bei weiterem ambulanten psychotherapeutischem Behandlungsbedarf nach vollstationärer multimodaler Schmerztherapie. Vor dem
Hintergrund, dass in der ambulanten Regelversorgung viele Schmerzpatient*innen lange Wartezeiten in Kauf nehmen müssen, bis sie einen Psychotherapieplatz erhalten [5], gibt es im CP VII die Möglichkeit, die stationären klinischen Erfolge in den häuslichen Alltag durch edukative und psychotherapeutische Methoden zu transferieren.

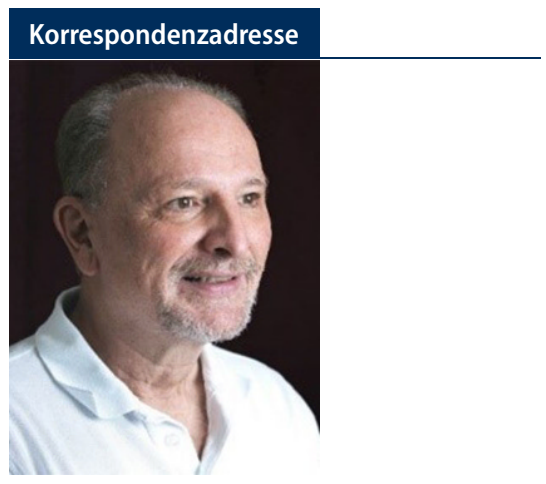

Dr. med. Hubertus Kayser

Abteilung Schmerzmedizin, Paracelsus-Klinik Bremen

In der Vahr 65, 28329 Bremen, Deutschland hubertus.kayser@pkd.de

\section{Einhaltung ethischer Richtlinien}

Interessenkonflikt. H. Kayser, N. Schneider und G. Schmiemann geben an, dass kein Interessenkonflikt besteht.

Für diesen Beitrag wurden von den Autoren keine Studien an Menschen oder Tieren durchgeführt. Für die aufgeführten Studien gelten die jeweils dort angegebenen ethischen Richtlinien.

\section{Literatur}

1. Berufsverband der Ärzte und Psychologischen Psychotherapeuten in der Schmerz- und Palliativmedizin in Deutschland e.V. (2019) BVSD-Weißbuch Schmerzmedizin. https://www.bvsd.de/ wp-content/uploads/2019/05/BVSD-Weissbuch Schmerzmedizin_2019_final.pdf Zugegriffen: 25 . Febr. 2021

2. Berufsverband der Ärzte und Psychologischen Psychotherapeuten in der Schmerz- und Palliativmedizin in Deutschland e.V. (2020) Umfrageergebnis des Berufsverbands der Ärzte und Psychologischen Psychotherapeuten in der Schmerz- und Palliativmedizin in Deutschland e. V. (BVSD). https://www.bvsd.de/bvsd-75-prozent-der-stationaeren-schmerzeinrichtungen-indeutschland-wegen-covid-19-geschlossen Zugegriffen:25. Febr. 2021

3. Bienek K, Marnitz U, Seidel W, Seifert C, von Pickardt B, Lindena G (2019) Interdisziplinär-multimodales sektorenübergreifendes Assessment und 
bedarfsorientierte Steuerung für Patienten mit Rückenschmerzen. Schmerz 33:116-127

4. Bundesärztekammer (BÄK), Kassenärztliche Bundesvereinigung (KBV), Arbeitsgemeinschaft der Wissenschaftlichen Medizinischen Fachgesellschaften (AWMF) (2017) Nationale VersorgungsLeitlinie Nicht-spezifischer Kreuzschmerz - Langfassung, 2. Auflage. Version 1. https://www. leitlinien.de/mdb/downloads/nvl/kreuzschmerz/ kreuzschmerz-2aufl-vers1-lang.pdf Zugegriffen: 25. Febr. 2021

5. Bundespsychotherapeutenkammer (2018) Ein Jahr nach der Reform der PsychotherapieRichtlinie - Wartezeiten 2018. https://www.bptk. de/wp-content/uploads/2019/01/20180411 bptk_studie_wartezeiten_2018.pdf Zugegriffen: 26. Febr. 2021

6. Casser H-R (2020) Schmerzmedizin in Zeiten von Corona:überflüssig? Orthop Rheuma 23(3):54

7. Deutsche Schmerzgesellschaft e. V. (2015) Handbuch DSF Deutscher Schmerz-Fragebogen. https://www.schmerzgesellschaft.de/fileadmin/ user_upload/DSF-Handbuch_2015.pdf Zugegriffen: 1. Nov. 2021

8. Dresler T, Guth A-L, Lüpke J, Kropp P (2020) Psychologische Kopfschmerztherapie in Zeiten von COVID-19. Schmerz 34:503-510

9. Hüppe $M$, Kükenshöner $S$, Böhme $K$, Bosse $F$, Casser H-R, Kohlmann T, Lindena G, Nagel B, Pfingsten M, Petzke F (2020) Schmerztherapeutische Versorgung in Deutschland - unterscheiden sich teilstationär versorgte Patienten von den ambulant oder stationär versorgten bei Behandlungsbeginn? Eine weitere Auswertung auf Basis des KEDOQ-Schmerz-Datensatzes. Schmerz 34:421-430

10. Institutfür Rehabilitationsmedizinische Forschung an der Universität Ulm (2021) Abschlussbericht Analyse der Daten des Modellprojekts „Caspar multimodal" der Deutschen Rentenversicherung Bund

11. Kaiser U, Petzke F, Nagel B, Marschall U, Casser H-R, Isenberg T, Kohlmann T, Lindena G, PAIN 2020 (2021) Evaluation eines frühen interdisziplinären multimodalen Assessments für Patienten mit Schmerzen. Schmerz 35:251-264

12. Kassenärztliche Bundesvereinigung (KBV) (2016) Vereinbarung von Qualitätssicherungsmaßnahmen nach § 135 Abs. 2 SGB V zur schmerztherapeutischen Versorgung chronisch schmerzkranken Menschen. https://www.kbv.de/media/sp/ Schmerztherapie.pdf Zugegriffen: 12. Okt. 2021

13. Kayser $H$, Thoma R, Mertens E, Sorgatz $H$, Zenz M, Lindena G (2008) Struktur der ambulanten Schmerztherapie in Deutschland. Schmerz 22:424-432

14. KayserH (2013) WirtschaftlicheDimensionen chronischer Rückenschmerzen. Bremer Ärztejournal 3/13:14-15

15. Lang E, Eisele R, Jankowsky H, Liebig K, Martus P, Neundörfer B (2000) Ergebnisqualität in der ambulanten Versorgung von Patienten mit chronischen Rückenschmerzen. Schmerz 14:146-159

16. Luchting B (2020) Interdisziplinäre multimodale Schmerztherapie unter den Schutzmaßnahmen der COVID-19-Pandemie. Schmerz 34:431-434

17. Neeb L, Ruscheweyh R, Dresler T (2020) Digitalisierung in der Kopfschmerzbehandlung. Schmerz 34:495-502

18. Pfingsten M, Arnold B, Böger A, Brinkschmidt T, Casser H-R, Irnich D, Kaiser U, Klimczyk K, Lutz J, Schiltenwolf M, Seeger D, Zernikow B, Sabatowski R (2019) Sektorenübergreifende

\section{Presentation of an innovative interdisciplinary and cross-sector therapeutic concept for pain patients as part of a regional selective contract}

Background: A graded therapeutic concept for the treatment of chronic pain patients in Germany is only available to a limited extent. Following the onset of coronavirus disease (COVID-19), care for these patients has become even worse.

Aim: To develop and establish a cross-sector therapeutic concept for chronic pain patients as part of a selective contract.

Methods: Embedded in existing therapeutic procedures, we define seven clinical pathways (CPs) into which patients are directed, after an interdisciplinary assessment according to refined criteria.

Organization: In CP I, patients remain in standard therapy. In CP II, patients have the opportunity to participate in an additional inter-profession education program. In CP III, patients get a specialized outpatient treatment. CP IV is a partial inpatient treatment, where multiple inpatient attendance days are replaced by tele-medical treatment, via a rehabilitation app. CP V and VI are inpatient treatments over 8 and 15 days each. If patients need further psychotherapeutic support after an inpatient treatment, they can be treated by clinical psychotherapists for another six months in CP VII.

Evaluation: The evaluation takes place 3, 6 and 12 months after initial assessment and includes the German Pain Questionnaire with different psychometric tests.

\section{Keywords}

Graded pain therapy concept · Interdisciplinary assessment $\cdot$ Partial inpatient treatment $\cdot$ Telemedical content treatment. Psychotherapeutic support

interdisziplinäre multimodale Schmerztherapie. Schmerz 33:191-203

19. Sabatowski R, Maier C, Willweber-Strumpf $A$, Thomm M, Nilges P, Kayser H, Casser R (2011) Empfehlung zur Klassifikation schmerztherapeutischer Einrichtungen in Deutschland. Schmerz 25:368-376

20. Shebib R, Bailey JF, Smittenaar P, Perez DA, Mecklenburg G, Hunter S (2019) Randomized controlled trial of a 12-week digital care program in improving low back pain. NPJ Digit Med 2:1. https://doi.org/10.1038/s41746-018-0076-7

21. SVR (Sachverständigenrat zur Begutachtung der Entwicklung im Gesundheitswesen) (2000/2001) Band III Rückenleiden. https://www.svrgesundheit.de/index.php?id=326 Zugegriffen: 25. Febr. 2021

22. SVR (Sachverständigenrat zur Begutachtung der Entwicklung im Gesundheitswesen) (2018) Bedarfsgerechte Steuerung der Gesundheitsversorgung.https://www.svr-gesundheit.de/fileadmin/ user_upload/Gutachten/2018/SVR-Gutachten_ 2018_WEBSEITE.pdf Zugegriffen: 25. Febr. 2021

23. Toelle TR, Utpadel-Fischler DA, Haas KK, Priebe JA (2019) App-based multidisciplinary back pain treatment versus combined physiotherapy plus online education: a randomized controlled trial. NPJ Digit Med 2:34. https://doi.org/10.1038/ s41746-019-0109-x

24. Wenig CM, Schmidt CO, Kohlmann T, Schweikert B (2009) Costs of back pain in Germany. Eur J Pain 13(3):280-286

25. Willweber-Strumpf A, Zenz M, Bartz D (2000) Epidemiologie chronischer Schmerzen. Schmerz 14:84-91

26. Wolff SV, Willburger $R$, Hallner D, Rusu AC, Rusche H, Schulte T, Hasenbring MI (2018) Avoidance-Endurance Fast-Screen (AE-FS). Schmerz 32:283-292 